\title{
Killip Class I
}

National Cancer Institute

\section{Source}

National Cancer Institute. Killip Class I. NCI Thesaurus. Code C77269.

A classification of myocardial infarction characterized by an absence of rales over the lung fields and an absence of S3. 